\section{Reactions of psychiatric patients to telepsychiatry}

\author{
Robbie Campbell, 1 Jennifer O'Gorman,2 \\ Zack Z. Cernovsky 1 \\ 1Lawson Health Research Institute and \\ Department of Psychiatry, University of \\ Western Ontario, London, ON; 2 London \\ Health Sciences Centre, London, ON, \\ Canada
}

\section{Abstract}

Telepsychiatry could offer a viable medical service to remote or isolated social communities if it does not generate adverse reactions such as delusional ideation, particularly in patients in settlements without adequate exposure to mainstream culture and internet. We examined subjective reactions to telepsychiatry of randomly selected 84 psychiatric patients from remote locations in Ontario, Canada. They rated the quality of their teleconferencing sessions via 10 item questionnaire and were asked about advantages and disadvantages of telepsychiatry. The majority of patients indicated that they were able to communicate as if physically present (92.9\%) and were comfortable with telepsychiatric service (95.2\%). They found the sessions as beneficial as direct meetings with their psychiatrist (84.5\%) and would use this service again (98.8\%). There were no instances of telepsychiatry being associated with adverse reactions in patients from remote communities with inadequate exposure to modern mainstream culture and internet.

\section{Introduction}

Psychiatric services to remote Canadian communities are obstructed by extreme distances or inclement weather conditions such as protracted snowstorms. By reducing the need for travel, telepsychiatry increases availability and speed of treatment for distant and chronically underserviced communities.1,2 Travel cost to remote areas of Canada is prohibitive. Telepsychiatry to distant communities is less expensive than the traditional mode of psychiatric contact, 3,4 and may be as effective across a wide variety of diagnostic categories, $5-7$ including schizophrenia. ${ }^{8}$ A satisfactory degree of diagnostic agreement was found in comparisons of direct contact and telepsychiatric interview. ${ }^{9}$ However, patients on remote, economically deprived, or culturally isolated locations rarely have adequate exposure to modern technology or access to internet. Their world-view may greatly differ from the mainstream culture. It is important to examine whether telepsychiatric contact has a potential of upsetting such patients or exacerbating their symptoms. Our study evaluated the patients' reactions to telepsychiatry in remote areas of Ontario, Canada.

\section{Materials and Methods}

A random survey was carried out with 84 psychiatric patients from various sites (including the First Nations) following their teleconferencing sessions, using a standard 10 item questionnaire. Specifically, they were asked to rate, on a 5 point scale, ranging from 1 (poor) to 5 (excellent), the quality of equipment, picture quality, the room environment, and also whether or not this session was helpful in addressing their problems. They were also asked to indicate whether or not they agreed or disagreed with the following 2 items: I was comfortable talking to the clinician using this type of service and I was able to communicate all information that needed presenting. They also were to respond, by choosing yes or no, to the following 2 items: I found this telepsychiatry session to be beneficial and Would you use this type of service again? Finally, they were to list the major benefits/disadvantages of telepsychiatry and to suggest what the health service providers could do to make this experience better. The study was approved by the local research ethics committee.

Prior to this study, our 84 participants had no access to a computer connected to internet or they lacked in sufficient expertise with communication programs such as Skype. Some of the remote Canadian communities had no internet access at all and no computers at the time. Our telepsychiatry equipment was the same on all remote sites and was provided via financial support by a government grant. All patients were personally visited by a psychiatrist prior to the installation of the equipment. Some lived at distances associated with excessive travel time or on locations inaccessible in certain winter conditions and this would preclude or obstruct a subsequent adequate medical contact with the patient, except via telepsychiatry. Many of our patients suffered from chronic or acute symptoms of psychosis in the form of schizophrenia, depression, or within the bipolar spectrum. The medication was delivered to patients by traveling nurses after the telepsychiatric interview or, if feasible, the patient had to travel to a pharmacy to receive the medication. Each of the patients with severe psychiatric symptoms obtained a frequent follow up, via telepsychiatry, as needed.
Correspondence: Zack Cernovsky, Department of Psychiatry, Faculty of Medicine, University of Western Ontario, London, ON, N6A4G5 Canada. Tel.: +1.519.471.8049 - Fax: +1.519.471.8049.

E-mail: zcernovs@uwo.ca

Key words: telepsychiatry; adverse events; future health technology.

Contributions: RC, design of the study, supervision of the research team, interviews with patients via telepsychiatry, preparation of the manuscript; JOG, interviews with patients via telepsychiatry, collection of questionnaire data, review of the manuscript; ZC, statistical analysis, preparation of the manuscript.

Conflict of interest: the authors declare no potential conflict of interest.

Funding: Canada Health Info Structure Partnerships Program (CHIPP) of Health Canada and the Ontario Ministry of Health and Long Term Care, Ontario, Canada.

Conference presentiation: this study is based on a paper presented in the Congress of the World Psychiatric Association, Prague, Czech Republic, October 17-21, 2012.

Received for publication: 6 July 2015 .

Revision received: 31 July 2015.

Accepted for publication: 4 August 2015.

This work is licensed under a Creative Commons Attribution NonCommercial 3.0 License (CC BYNC 3.0).

(C) Copyright R. Campbell et al., 2015

Licensee PAGEPress, Italy

Mental Illness 2015; 7:6101

doi:10.4081/mi.2015.6101

No demographic and diagnostic information was compiled on these participants, in order to safeguard their anonymity and to provide an opportunity for a free expression of feelings about telepsychiatry without concern about negative consequences for their relationship to psychiatric staff.

\section{Results}

The majority $(97.6 \%)$ of the patients rated the sound quality as good to excellent and $95.2 \%$ gave similar ratings to picture quality. Furthermore, $95.2 \%$ of the patients indicated by their ratings that they were comfortable with telepsychiatry service, $92.9 \%$ that they were able to communicate adequately, $84.5 \%$ of the patients found the sessions as beneficial as a direct physical presence, and $98.8 \%$ reported that they would use the service again. 
It is noteworthy that only one of the 84 patients found telepsychiatry stressful. There were no reported instances where telepsychiatry caused any escalation of delusional or of other psychiatric symptoms.

\section{Discussion and Conclusions}

While the absence of demographic, diagnostic, and follow up data constitutes a methodological limitation, the patients' anonymity in these respects prevents that a negative rating of telepsychiatry by a patient could be followed by negative consequences for subsequent therapeutic contacts.

The significance of this study for public health is as follows. Our survey indicates that almost all patients were satisfied with the current technical status of telepsychiatry and felt comfortable with the telepsychiatry as a mode of contact. The patients did not need to travel extreme distances or in dangerous weather conditions to obtain service and they benefited from decreased waiting time for appointments. In too many remote or underserviced regions of our world, telepsychiatry is the only current- ly viable (and often also a life-saving) option for emotionally distressed persons to receive a psychiatric evaluation or psychological therapy.

\section{References}

1. Hilty DM, Marks SL, Urness D, et al. Clinical and educational telepsychiatry applications: a review. Can J Psychiatry 2004;49: 12-23.

2. Urness D, Weisser L, Campbell R, Hilty D. Telepsychiatry in Canada and the United States. In: Wooton R, Yellowlees P, McLaren P, eds. Telepsychiatry and e-mental health. London: The Royal Society of Medicine Press; 2003. pp 97-111.

3. 0'Reilly RL. The costs of providing psychiatric consultation and follow up in person versus via videoconference: the results of a randomized control trial. Paper presented at the Annual Meeting of the Canadian Psychiatric Association, October 30November 2, 2003. Halifax, Nova Scotia, Canada.

4. Trott P, Blignault I. Cost evaluation of a telepsychiatry service in northern Queensland. J Telemed Telecare 1998;4: 66-8.

5. Kennedy C, Yellowlees P. The effectiveness of telepsychiatry measured using the Health of the Nation Outcome Scale and the Mental Health Inventory. J Telemed Telecare 2003;9:12-6.

6. Ruskin PE, Silver-Aylaian M, Kling MA, et al. Treatment outcomes in depression: comparison of remote treatment through telepsychiatry to in-person treatment. Am J Psychiatry 2004;161;1471-6.

7. Hilty DM, Ferrer DC, Parish MB, et al. The Effectiveness of telemental health: a 2013 review. Telemed J E-health 2013;19:44454.

8. Kasckow J, Felmet K, Appelt C, et al. Telepsychiatry in the assessment and treatment of schizophrenia. Clin Schizophr Relat Psychoses 2014;8:21-27A.

9. Seidel RW, Kilgus MD. Agreement between telepsychiatry assessment and face-to-face assessment for Emergency Department psychiatry patients. J Telemed Telecare 2014;20:59-62. 